\title{
Stellar winds and the circumstellar environment of massive first stars
}

\author{
Jiří Krtička ${ }^{1}$ and Jiří Kubát ${ }^{2}$ \\ ${ }^{1}$ Masarykova univerzita, CZ-611 37 Brno, Czech Republic, \\ email: krticka@physics.muni.cz \\ ${ }^{2}$ Astronomický ústav, CZ-251 65 Ondřejov, Czech Republic
}

\begin{abstract}
We study dynamical aspects of circumstellar environment around massive first stars. We show that the stellar winds from stationary massive first generation (Population III) stars driven either by the line or continuum transitions are unlikely. Massive first stars are also unable to expel any element or isotope with a possible exception of ${ }_{1}^{1} \mathrm{H}$. H-He stars with Eddington parameter $\Gamma \gtrsim 0.859$ may have pure-hydrogen wind with mass-loss rates of order $10^{-14} \mathrm{M}_{\odot}$ year ${ }^{-1}$. Finally, we show that hydrogen and helium lines could be important for shutting off the initial accretion onto first stars and may influence the initial mass function of first stars.
\end{abstract}

Keywords. Hydrodynamics, stars: mass loss, stars: winds, outflows

\section{Introduction}

The Big Bang nucleosynthesis produced a metal-free Universe. Thus, the very first stellar generation consisted of initially pure hydrogen and helium stars. The outflows from first stars have not been systematically studied up to now. To fill this gap we present the discussion of pure $\mathrm{H}-\mathrm{He}$ line-driven winds.

\section{Wind models}

For the numerical tests we used NLTE wind models described by Krtička \& Kubát (2004). The computer program allows to solve hydrodynamic, simplified radiative transfer, and statistical equilibrium equations in a radiatively driven stellar wind. For the present purpose we slightly changed the code. Since we do not know in advance whether the stellar wind is possible or not, the hydrodynamical variables, i.e. velocities, temperatures, and densities of all wind components (with an exception of the electron density) are kept fixed. This enables us to calculate the model occupation numbers and the radiative force regardless of the existence of the wind and, consequently, to test the wind existence.

The necessary condition for launching the stellar wind has the form of $g^{\text {rad }}>g$, i.e. the total (line and continuum) radiative acceleration $g^{\text {rad }}$ at some point in the wind has to be larger than the gravity acceleration $g$. The line radiative force is calculated in the Sobolev approximation after Castor (1974). We assume zero rotational velocity.

Stellar radiative flux is taken from static spherically symmetric model stellar atmospheres calculated using NLTE code of Kubát (2003). The radiative force in these models was calculated without any simplification. The total radiative force in all these model stellar atmospheres is always lower than gravity.

Parameters of studied hot supermassive stars are taken from the list of Kudritzki (2002). For each of these stars three models with different values of tentative mass-loss rates were calculated, $10^{-6} \mathrm{M}_{\odot}$ year ${ }^{-1}, 10^{-7} \mathrm{M}_{\odot}$ year $^{-1}, 10^{-8} \mathrm{M}_{\odot}$ year $^{-1}$. 


\section{Calculated models}

Hydrogen-helium models: For studied model stars we tested whether the radiative force due to $\mathrm{H}$ and $\mathrm{He}$ lines is able to drive stellar wind. Due to low occupation numbers of bound states of $\mathrm{H}$ and $\mathrm{He}$ the radiative force in all our models is lower than the gravity force. Also the bound-free and free-free radiative force is negligible. We conclude that hot massive first-generation stars fail to drive $\mathrm{H}-\mathrm{He}$ wind.

Influence of minor isotopes: To test importance of isotopes ${ }_{1}^{2} \mathrm{H},{ }_{2}^{3} \mathrm{He}$ and lithium we included it into our atomic list. Our models show that their contribution to the radiative force is negligible.

Expulsion of individual elements: Whereas first stars fail to drive H-He outflow, there is a possibility that $\mathrm{H}$ or $\mathrm{He}$ are expelled separately, i.e. that there exists a pure $\mathrm{H}$ or $\mathrm{He}$ wind. Whereas the radiative force in $\mathrm{He}$ wind is too low to expel He out from the stellar atmosphere, $\mathrm{H}-\mathrm{He}$ stars close to the Eddington limit with $0.859 \lesssim \Gamma<1$ may have pure $\mathrm{H}$ wind driven only by the light scattering on free electrons (with mass-loss rate of order $\dot{M} \approx 10^{-14} \mathrm{M}_{\odot}$ year $^{-1}$ ). Finally, expulsion of other isotopes or elements (i.e. ${ }_{1}^{2} \mathrm{H}$, ${ }_{2}^{3} \mathrm{He}$ and lithium) is unlikely.

Line-transitions and accretion: We have also tested whether the line radiative force is able to terminate the initial accretion onto first stars. We have found that this is possible only for stars relatively close to the Eddington limit and with relatively small accretion rates $\dot{M} \lesssim 10^{-5} \mathrm{M}_{\odot}$ year ${ }^{-1}$. Consequently, it is possible that for massive stars the radiative force can influence the accretion physics and the initial mass-function of first stars. More detailed description of our models and results can be found elsewhere (Krtička \& Kubát 2005).

\section{Mass-loss and evolutionary models}

A general picture of stellar wind properties of low-metallicity massive stars emerges:

i. Hot massive zero-metallicity stars with $\Gamma \lesssim 0.859$ do not have any stellar wind. If their atmospheres are relatively quiet the chemical peculiarity may develop there. H-He stars with Eddington parameter $\Gamma \gtrsim 0.859$ may have a pure-hydrogen wind.

ii. For extremely low metallicities pure metallic line-driven wind may develop.

iii. For higher metallicities also $\mathrm{H}$ and $\mathrm{He}$ are expelled from the atmosphere and multicomponent effects become important in such stellar wind (Krtička et al. Krtička et al. 2003).

iv. For even higher metallicities a present-day line-driven wind exist.

The best choice for evolutionary calculations is to assume zero mass-loss rate for very low-metallicity stars i.-ii. and to use Kudritzki (2002) prescription for other stars.

\section{Acknowledgements}

This work was supported by grants GA ČR 205/03/D020, 205/04/1267. JKr is grateful to IAU for a travel grant.

\section{References}

Castor, J. I. 1974, MNRAS 169, 279

Krtička, J., \& Kubát, J. 2004, A\&\&A 417, 1003

Krtička, J., \& Kubát, J. 2005, $A \& A$, submitted

Krtička, J., Owocki, S. P., Kubát, J., Galloway, R. K., \& Brown, J. C. 2003, A $\& A$ 402, 713

Kubát, J. 2003, IAU Symp. 210, A8

Kudritzki, R. P. 2002, ApJ 577, 389 